\title{
Experimental Study on Thermal Vacuum Environment Sensitivity of Spacecraft Antenna's Typical Failure
}

\author{
Bi Yanqianga, WangJing, QieDianfu,Shang Yonghong and Li Xiyuan \\ Beijing Institute of Spacecraft Environment Engineering, Beijing, China
}

\begin{abstract}
With the development of space applications, spacecraft antenna has become an indispensable part of any space system. The spacecraft antenna affects and constrains the performance and functionality of the entire wireless communication system as well as the entire spacecraft. Spacecraft antenna has to withstand the noise, vibration, shock and acceleration as launched, and weightlessness, high vacuum, radiation, extreme hot and cold alternating space environment on-orbit[1].The influence of different environmental factors on the typical failure modes of spacecraft antenna is different. The environmental adaptability of the spacecraft antenna depends mainly on its structural design, material, process and other factors. In this paper, the influence of different environmental factors on the typical failure modes of the spacecraft antenna is studied. The sensitivity analysis of the typical failure modes of the thermal vacuum environment is verified by experiments, which provides support for the development of the spacecraft antenna.
\end{abstract}

\section{Introduction}

Antenna Thermal Vacuum Environment Fault Mode Sensitivity refers to the degree of influence of different thermal environmental factors such as vacuum, high and low temperature, temperature rise and fall rate, and number of thermal cycles on the antenna failure. An environmental factor is likely to cause thermal failure mode of the antenna. This indicates that the factor influence degree is high, and this failure mode is highly sensitive to this environmental factor[2].The research process is based on the results of the failure model study and analyzes the results of changes in environmental factors.

The influence of each environmental factor on each failure mode is analyzed, and the sensitivity of each failure mode to each thermal vacuum environment is divided into three grades: high, medium and low according to the degree of influence. Certain change of environmental factors can induce a fault, and the environmental factors sensitive to the failure is high; If an environmental factor to be combined with other environmental factors induce a failure, the environmental factor is middle sensitivity to the failure; An environmental factor in normal range in any case will not induce a certain type of failure, then determine this environmental factor is low sensitivity to the failure.

Antenna structure in the hot environment is prone to skin delamination, tear, surface accuracy deterioration, gas out, circuit abnormalities and other failures. In this paper, the sensitivity of thermal environment factor to failure mode is analyzed and verified by experiments.

\section{Thermal Vacuum Environment Sensitivity Analysis to Typical Fault}

\footnotetext{
a Corresponding author: biyq05@mails.tsinghua.edu.cn
} 
Thermal vacuum environmental factors include vacuum, high temperature, low temperature, number of cycles, cooling rate and so on. The sensitivity of the degree of vacuum, high and low temperature, and cycle times to typical faults is analyzed separately.

\subsection{The vacuum sensitivity}

One of the reasons for the occurrence of antenna reflector skin bag is the residual gas inside honeycomb structure and poor ventilation, and there is pressure difference inside and outside the honeycomb under vacuum conditions. Assuming aluminum honeycomb side length $L=6 \mathrm{~mm}$, high $h=15 \mathrm{~mm}$ as shown in Figure 1, aluminum film thickness $d_{\mathrm{L}}=0.03 \mathrm{~mm}$, so under low pressure $p$ the pressure difference $\Delta p=p_{0}$ - $p$ and $p_{0}$ is initial pressure inside honeycomb. The stress between the skin and honeycomb is $\mathrm{s}=\sqrt{3 / 4} \mathrm{LDp} / \mathrm{d}_{\mathrm{L}}$.
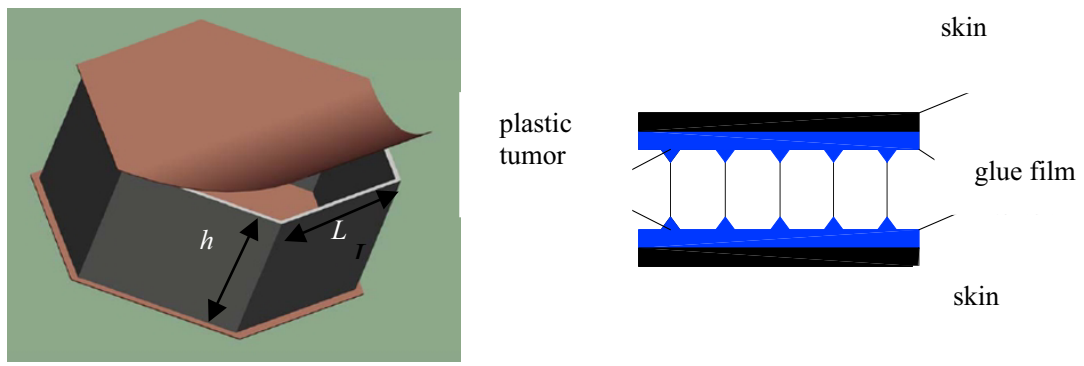

Figure 1.Aluminum honeycomb unit

When the pressure $\mathrm{p}$ is $10^{-3} \mathrm{~Pa}, \mathrm{~s}=17 \mathrm{MPa}$,close to tensile strength of RTV glue. So vent hole is added in processing technology, the other method is increase the size of tumor appropriately. Figure 2 shows tensile stress of structural adhesive with size changes of tumor under different degree of vacuum, increasing size of tumor can significantly reduce stress inside the structure.
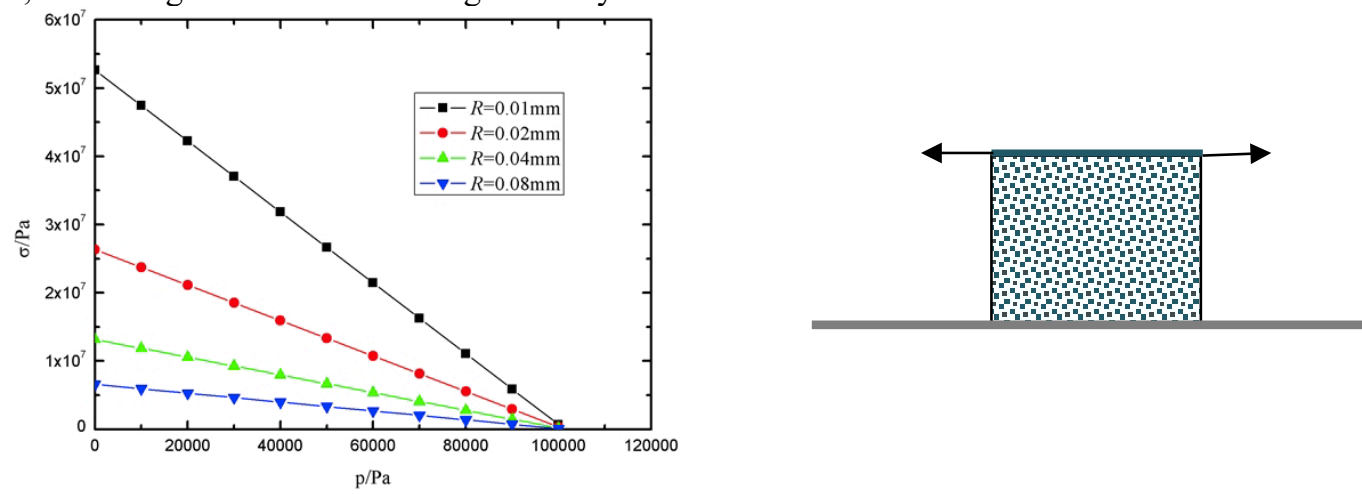

Figure 2.Effect of vacuum on stress under different size Figure 3.Vacuum degree on skin

The stress on skin generated by gas in honeycomb is shown in Figure 3. When the maximum vacuum is $0 \mathrm{~Pa}$, the maximum tensile stress of skin is $s=p h / d$, where $P$ is atmospheric pressure, $d$ is thickness of skin and structural adhesive and take $0.5 \mathrm{~mm}$. The maximum tensile stress of skin is $3 \mathrm{MPa}$, which is much smaller than the allowable stress of material $295 \mathrm{MPa}$. Therefore, in the case of other environmental conditions unchanged, the vacuum is not enough to cause the antenna skin tear phenomenon, so the sensitivity of vacuum to skin tear is low.

\subsection{High, low temperature sensitivity}

The cell's temperature is higher, the pressure would be greater. Assuming pressure in honeycomb is 
$P_{0}$ at atmospheric temperature $T_{0}$, the pressure in honeycomb would be $P=P_{0} T / T_{0}$ when temperature rises to $T$. In sealed honeycomb core, tensile stress of structural adhesive between skin and aluminum foil changes with temperature to $\sigma=\frac{\sqrt{3} L}{2 d}\left(\frac{T-T_{0}}{T_{0}}\right) P_{0}$. Figure 4 shows stress changes with temperature under different tumor size.

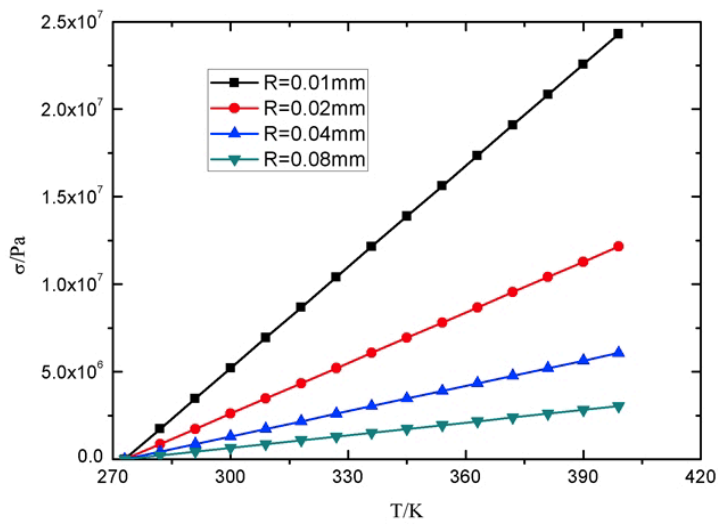

Figure 4.Effects of temperature on stress under different size

In addition after temperature is changed, thermally stress would be exist between inner and outer layers of antenna skin due to difference of thermal expansion. When thermal stress of outer layer is greater than allowable stress, skin will be damaged. The physical model of tear failure mode is shown as Figure 5.In order to analyze rationality and convenience, following assumptions were made for analysis model. 1)Initial temperature is ambient atmospheric temperature, and no internal stress;2)Ignoring the effect of inner layer gas-out of the antenna on tearing of the skin;3)The curvature of the antenna skin is zero; 4) Temperature of each layers is the same.

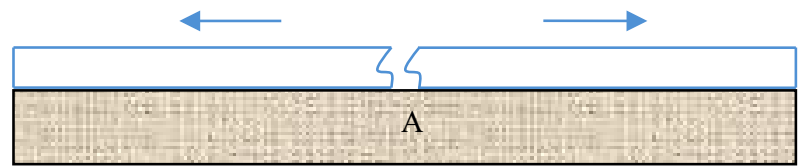

Figure 5.Skin tear analysis physical model

The mathematical model was established based on the previous assumptions and physical model, and simplified as shown below,

$$
\left\{\begin{array}{l}
\Delta L_{\mathrm{A}}=\beta_{\mathrm{A}} \cdot\left(T-T_{0}\right), \Delta L_{\mathrm{B}}=\beta_{\mathrm{B}} \cdot\left(T-T_{0}\right) \\
\delta=\left(\Delta L_{\mathrm{A}}-\Delta L_{B}\right) / 2, \sigma_{T}=\delta \cdot E
\end{array}\right.
$$

So $\sigma_{T}=\left[\beta_{A}\left(T-T_{0}\right)-\beta_{B}\left(T-T_{0}\right)\right] E / 2$, Where $\beta_{A}$ and $\beta_{B}$ are the thermal expansion coefficients of layer $A$ and $B$ respectively. $\sigma_{\mathrm{T}}$ is the stress of layer B material. When the stress of B layer is greater than its allowable stress, tearing phenomenon will occur. Through analyze of effect of the temperature on the skin tear, the higher the temperature the higher the tensile stress of the skin, and the temperature continues to rise there is a tendency of tear on skin.

Therefore, high temperature is sensitive and low temperature is insensitive to skin tear failure mode.

\subsection{The number of cycles sensitivity}

In actual flight of spacecraft, material and structure would be suffered thousands of times alternating stress[3]. Ground thermal cycles can whether expose fatigue failure of antenna skin was analyzed.

If structure is absence of defects, fatigue failure is mainly related to stress amplitude, stress ratio, 
cycle period and number of cycles. In thermal test corresponding to test conditions are: high and low temperature difference, high and low temperature retention time, number of cycles. The maximum stress at which the infinite stress cycle occurs without breaking occurs is the fatigue limit. The antenna design should meet design requirements, which the stress is lower than fatigue limit. The relationship between the maximum stress and the number of cycles at which the failure occurs can be expressed as the $S-N$ curve. Material $S-N$ relationship expression is generally $S^{\mathrm{m}} N=\mathrm{C}$. Where $\mathrm{m}$ and $\mathrm{C}$ are the material-related constants[4].General rubber materials' $\mathrm{m}$ and Care taken 2 and $10^{12}$.Figure 6 shows the relationship between the maximum stress and number of cycles of glue material.

The design will be checked whether fatigue limit is met. That is, stress of material is below the straight line in Figure 6.As can be seen from Figure6, even if material stress is subjected to a 10-fold increase, it is necessary to undergo nearly a thousand thermal cycles to expose fatigue failure. Average number of cycles of the antenna thermal test is only 10 times, and far from the number to exposed fatigue failure. Thus, the number of cycles is less sensitive to the failure.

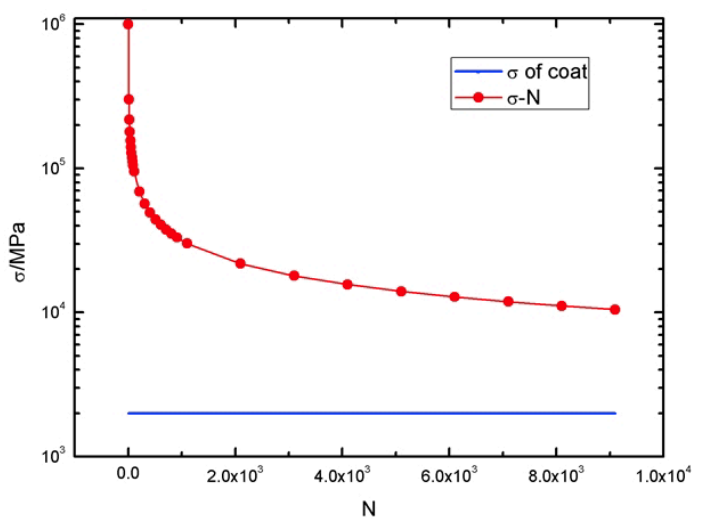

Figure 6.The relationship between the maximum stress value and the number of cycles

\section{Experimental verification}

\subsection{Experimental design}

According to the level of sensitivity and experimental conditions, the experimental and experimental environment were designed[5,6]. The experiment was conducted through the following stages:

1) Vacuum section, the specimen to withstand only vacuum effect;

2) Low temperature section, the specimen to withstand vacuum and low temperature;

3) Narrow temperature range, that is, small high temperature and low temperature;

4) Very low temperature, the specimen to withstand very low temperature;

5) High temperature, the temperature from $80^{\circ} \mathrm{C}$, heated interval $20^{\circ} \mathrm{C}$, up to $160^{\circ} \mathrm{C}$.

\subsection{Experimental results}

The experimental process obtained the antenna temperature, container vacuum degree, respectively is shown in Figure 7 and Figure 8. The antenna specimen before experiment is shown as Figure 9 and after experiment as Figure 10.During experiment the antenna's skin was damaged. 


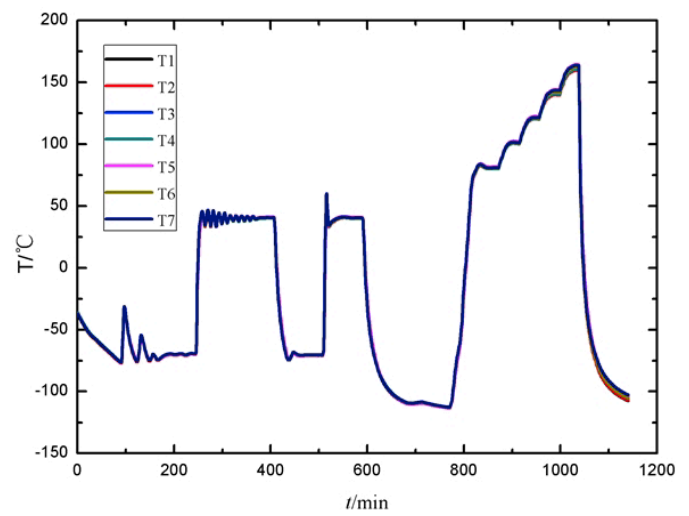

Figure 7.The temperature changes with time

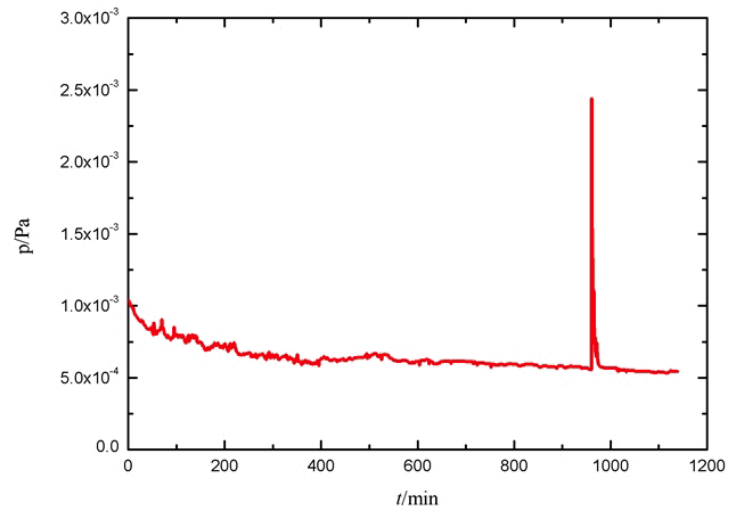

Figure 8. Vacuum degree changes with time

During the process of experiment, only the temperature reached $133^{\circ} \mathrm{C}$, the vacuum degree curve appeared a peak jump, indicating that at this time there was a gas release in container. The specimen was destruction at this time. The results of sensitivity analysis show that high temperature and vacuum have higher influence on the delamination of antenna skin. In the process of verification, the combination of vacuum and high temperature conditions leads to the occurrence of fault, which verifies the correctness of fault analysis and provides support for the following test conditions design.
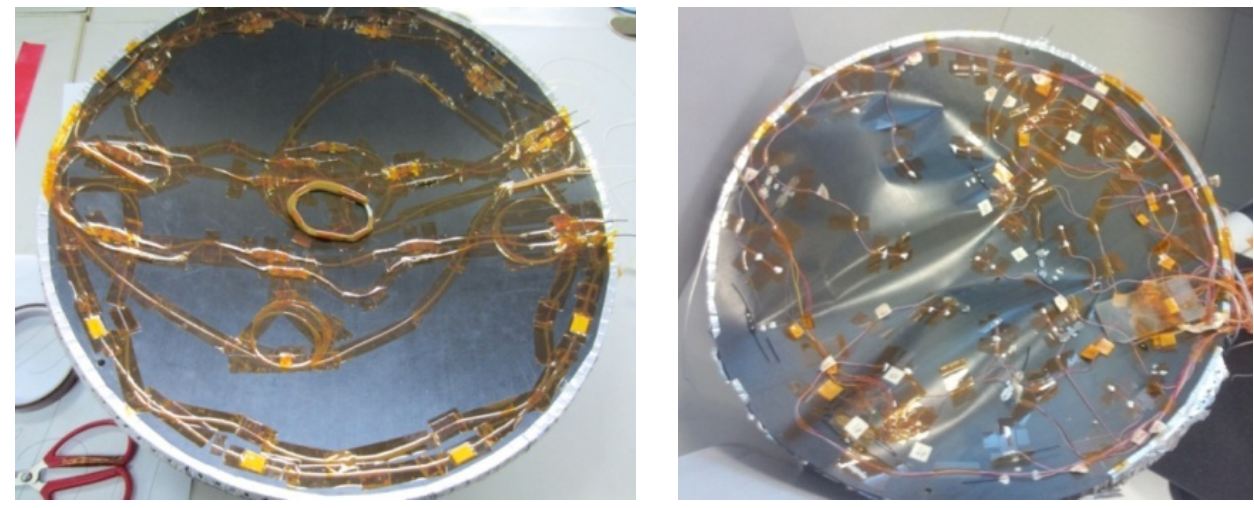

Figure 9.Antenna specimen before experiment Figure 10. Antenna specimen after experiment

\section{Conclusion}

Through the analysis, it is found that the spacecraft antenna has high sensitivity to high temperature and vacuum environment, and has low sensitivity to low temperature and cycle times. Through a reasonable experimental design, low temperature, cycle heating and other stress were put on the antenna firstly, no damage was found. Then high temperature, vacuum and other stress were put on the antenna, vacuum degree inside the test chamber decreased because of the antenna's gas-out. The antenna was damaged. Sensitivity of the antenna to the high temperature vacuum environment is verified by experiments. Based on the research results, it is possible to design the antenna thermal vacuum test reasonably, evaluate the reliability of the antenna design and ensure the performance of the spacecraft.

\section{References}

1. Ye Yunshang. Spacecraft Antenna Beijing: China Science and Technology Press, 2008. 
2. Ghalaty, N, F.; Aysu,A.; Schaumont, P. Analyzing and eliminating the causes of fault sensitivity analysis. Design, Automation an Test in Europe Conference and Exhibition(DATE), 2014.

3. Paden, R. S. Choosing thermal cyclic screening requirements to achieve desired test effectiveness. Proceedings of the 21st Aerospace Testing Seminar, Manhattan Beach, CA, 2003.

4. Fan Qinshan, Yin Yajun, Tan Jinglin. Mechanics of Materials. Beijing: High Education Press, 2014.

5. Huang Bencheng, Ma Youli. Space Environment Test Technology of Spacecraft. Beijing: National Defense Industry Press, 2002.

6. Welch, J. W. Unit thermal vacuum test consideration. Spacecraft Thermal Control Technology Workshop, The Aerospace Corporation, Feb. 2001. 